Article

\title{
Diet and Nutraceutical Supplementation in Dyslipidemic Patients: First Results of an Italian Single Center Real-World Retrospective Analysis
}

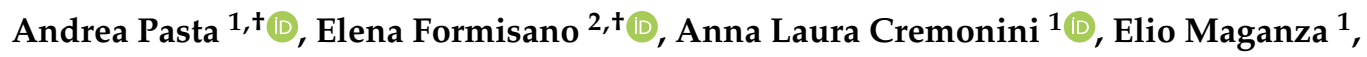 \\ Erika Parodi ${ }^{1}$, Sabrina Piras ${ }^{1}$ and Livia Pisciotta ${ }^{1,3, *}$ \\ 1 Department of Internal Medicine, University of Genoa, 16132 Genoa, Italy; andreapasta93@gmail.com (A.P.); \\ annalauracremonini@gmail.com (A.L.C.); elio.maganza@gmail.com (E.M.); erika-parodi@alice.it (E.P.); \\ piras.sabri@tiscali.it (S.P.) \\ 2 Nutritional Unit ASL-1 Imperiese, Giovanni Borea Civil Hospital, 18038 Sanremo, Italy; \\ e.formisano@asl1.liguria.it \\ 3 Dietetics and Clinical Nutrition Unit, IRCCS Policlinic Hospital San Martino, 16132 Genoa, Italy \\ * Correspondence: livia.pisciotta@unige.it; Tel.: +39-0103-538-689 \\ + These authors equally contributed to this work.
}

Received: 18 June 2020; Accepted: 8 July 2020; Published: 10 July 2020

check for updates

\begin{abstract}
Background: Dyslipidemias are a heterogeneous group of metabolic disorders mainly characterized by an increased risk of atherosclerotic cardiovascular disease (ASCVD) or other conditions, such as acute pancreatitis in hypertriglyceridemia. The aim of this study was to evaluate the effect of diet treatment and nutraceutical (NUTs) supplementation on the plasma lipid profile in outpatient dyslipidemic subjects, considering the influence of several factors (i.e., gender, age, body mass index, alcohol consumption, and smoking habits). Methods: 487 dyslipidemic patients spanning from 2015 to 2019 were treated with a Mediterranean diet or NUTs in a real-word setting and were retrospectively analyzed. General characteristics and lipid profile at baseline and after the follow-up period were evaluated. Results: Diet alone reduced total cholesterol (-19 mg/dL, -7.7\%), LDL cholesterol (-18 mg/dL, -10.1\%), and triglycerides (-20 mg/dL, $-16.7 \%)$. Triglycerides (TG) decreased more in men, while women were associated with higher reduction of LDL cholesterol (LDL-C). Different types of NUTs further ameliorate lipid profiles when associated with diet. Nevertheless, most patients at low ASCVD risk (222 out of 262, 81.6\%) did not achieve the 2019 ESC/EAS guidelines recommended LDL-C goals (i.e., LDL-C < $116 \mathrm{mg} / \mathrm{dL}$ ). Conclusion: Lipid-lowering diet improves lipid profile, and NUTs can boost its efficacy, but taken together they are mainly unsatisfactory with respect to the targets imposed by 2019 EAS/ESC guidelines.
\end{abstract}

Keywords: lipid lowering diet; nutraceutical supplements; dyslipidemias; hypercholesterolemia; hypertriglyceridemia; EAS/ESC guidelines LDL-C targets

\section{Introduction}

Dyslipidemias are a heterogeneous group of metabolic disorders mainly characterized by an increased risk of atherosclerotic cardiovascular disease (ASCVD) [1-3]. The most common phenotypes of hyperlipidemias are hypercholesterolemia, characterized by high levels of serum cholesterol due to high low-density lipoprotein cholesterol (LDL-C) levels, hypertriglyceridemia, caused by high levels of triglycerides (TG), and mixed hyperlipidemia, which refers to both elevated LDL-C and plasma TG [4]. Severe hypertriglyceridemia (TG $>1000 \mathrm{mg} / \mathrm{dL}$ ) is associated with increased risk of acute pancreatitis [5]. Hypoalphalipoproteinemia is directly related to the risk of coronary heart disease [6]. 
To date the pharmacological treatment of hyperlipidemias involves various types of drugs: statins (inhibitors of cholesterol synthesis) have fully demonstrated efficacy in improving the lipid profile and reducing cardiovascular risk [7], however, LDL-C serum levels achieved with statins do not fully reduce the risk of ASCVD [8]. These drugs can be associated with ezetimibe (inhibitor of cholesterol intestinal absorption) $[9,10]$ or to innovative biological therapies such as monoclonal antibodies [11]. Fibrates are also used in lipid-lowering therapies, especially in patients with high levels of triglycerides [12].

The guidelines for the management of dyslipidemias $[13,14]$ support the role of lifestyle change as a basic treatment. Lifestyle treatment involves a diet based on the Mediterranean model, low in saturated fat and cholesterol and rich in whole grains, vegetables, fruits, and increased physical activity $[15,16]$. Patients with hypertriglyceridemia should also reduce sugar intake and avoid alcohol consumption [17]. Most evidence has shown that diet treatment has a low and variable effect on serum LDL-C, in a range of $5-15 \%$ [18-21].

Consequently, in clinical practice, the use of diet alone for the treatment of dyslipidemias could be unsatisfactory. In fact, in low- and moderate-risk patients, the 2016 ESC/EAS guidelines [13] recommend LDL-C levels less than $115 \mathrm{mg} / \mathrm{dL}$, but the new $2019 \mathrm{ESC} / \mathrm{EAS}$ guidelines [14] recommended LDL-C $<116 \mathrm{mg} / \mathrm{dL}$ and $<100 \mathrm{mg} / \mathrm{dL}$ in low- and moderate-risk patients, respectively.

As a part of lifestyle modifications, the use of lipid lowering nutraceuticals (NUTs) can be considered alone or in combination with diet and provide a moderate reduction in blood cholesterol [14]. Lipid lowering nutraceuticals are natural dietary supplements which can be used to improve the lipid profile in low ASCVD risk patients and subjects with statin intolerance [22,23]. These compounds act in different ways: monacolin $\mathrm{K}$ is an inhibitor of hepatic cholesterol biosynthesis, berberine acts as a promoter of cholesterol uptake in the liver inhibiting the proprotein convertase subtilisin/kexin type 9 (PCSK9) and plant sterols are inhibitors of intestinal cholesterol absorption [24]. Other class of nutraceuticals are represented by Fatty acids- $\omega 3$, which are successfully used in the treatment of hypertriglyceridemia [25-27].

To date, the factors which could influence the success of a lifestyle intervention with diet alone or supplemented with NUTs are still objects of investigation. Furthermore, an open finding is the contextualization of the improvement of the lipid profile, and in particular of the LDL-C, in the recommendations of the ESC/EAS guidelines for the management of dyslipidemias. Thus, the present study aims to evaluate the effect of diet treatment and NUTs on plasma lipid parameters, and how specific factors (i.e., gender, age, body mass index, alcohol consumption and smoking habits) could influence lipid profile and lifestyle modifications in a real-world setting considering the targets of ESC/EAS guidelines as references.

\section{Materials and Methods}

\subsection{Subjects}

The present study is a retrospective analysis of 2472 patients' medical records affected by different types of dyslipidemias and referred to the outpatient section of the Lipid Clinic of IRCCS Policlinic San Martino Hospital, University of Genoa, Italy, from 2015 to 2019.

At baseline, all subjects underwent a medical evaluation: family, remote, and near pathological history were considered, and body mass index (BMI) and arterial pressure were assessed. Complete lipid profile, measured in the absence of therapy, was reported (total cholesterol, high-density lipoprotein cholesterol, and TG). A specialized physician with extensive experience in the management of dyslipidemias stratified all patients according to their cardiovascular risk through SCORE algorithm or diagnosing a pathological condition, such as familial hypercholesterolemia, familial combined hyperlipidemia, or other high cardiovascular risk related conditions. The therapeutic strategies were formulated according to the guidelines' recommendations $[13,14]$ and the rules of the Italian Ministry of Health [28]. Thus, the treatments included lifestyle modifications (i.e., diet and nutraceutical supplements, loss of weight, physical activity, smoking discontinuation) associated or not with 
lipid-lowering drug therapy. All subjects were expected to return a few months later and underwent a follow-up visit to evaluate the effects of the therapy on biochemical tests. The compliance to the lifestyle intervention was assessed during the follow-up visit by asking the patients.

In this retrospective study, we focused our formal analysis on 487 patients treated only with lifestyle modifications coming back at the follow-up visit. The flow chart in Figure 1 reports the allocation criteria and grouping for patients' analysis as previously described.

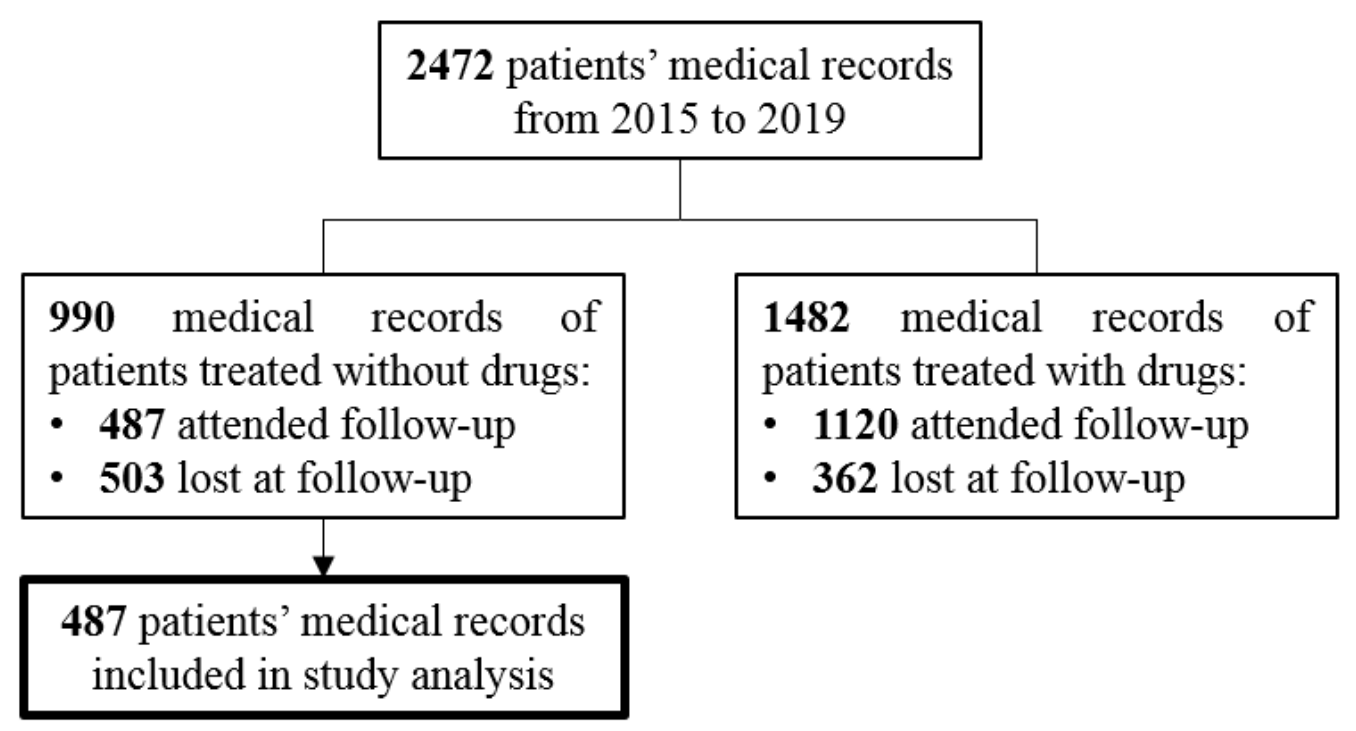

Figure 1. Flow chart of study design and allocation criteria and grouping for patients' former analysis.

Informed written consent for using personal data for the present investigation was obtained from all the subjects. The study was conducted in accordance with the Declaration of Helsinki and was approved by the Ethics Committee of IRCCS Policlinic Hospital San Martino in Genoa (Italy) (Project number 270/2020).

\subsection{Data Collection and Ranking Methods}

Blood tests performed in an authorized laboratory within four weeks since baseline were evaluated and total cholesterol (TC), high-density lipoprotein cholesterol (HDL-C) and TG were retrospectively registered. LDL-C was calculated by the Friedewald formula. The same data were collected at follow-up.

Gender, age, systolic and diastolic blood pression (SBP, DBP), follow-up period, weight, height, and BMI were registered. Patients were classified in no/past smokers and current smokers [29]. Alcohol intake was collected as follow: no/moderate consumption if lower than $10 \mathrm{~g} / \mathrm{day}$ and excessive consumption if higher than $10 \mathrm{~g} /$ day [14]. BMI and age of patients were binary ranked in normal or overweight [30] and in younger or older than 45 years [31], respectively.

\subsection{Lifestyle Intervention}

According to the ESC/EAS guidelines for the management of dyslipidemias [13,14], a lipid lowering diet, based on the Mediterranean model, was administered in all patients and was characterized by a lipid intake between 25 and $35 \%$ of the daily kcal, with saturated fats $<7 \%$ of the total kcal and cholesterol lower than $300 \mathrm{mg} /$ day. The carbohydrate and protein intakes were $45-55 \%$ and $15-25 \%$ of the total daily kcal, respectively. Patients were also advised to do regular moderate-intensity exercise ( $\geq 30$ min a day) for both normal-weight and overweight.

After the evaluation of anthropometric and hematochemical parameters, four different types of diets were administered in an outpatient setting: 
- General advice to reduce the excess of saturated fats and cholesterol intake based on weekly food frequency in normal weight patients with primary hypercholesterolemia

- General advice to reduce the excess of carbohydrates and alcohol based on weekly food frequency in normal weight patients with hypertriglyceridemia or mixed hyperlipemia

- Diet with weekly food frequency and total energy intake of $1700 \mathrm{kcal} /$ day in overweight women

- Diet with weekly food frequency and total energy intake of $2100 \mathrm{kcal} /$ day in overweight men.

NUTs were administered in some subjects according to guidelines: Monacolin K (MonK), Phytosterols (PS), Berberine (BBR), Omega-3 Polyunsaturated Fatty Acids (PUFA-W3) and associations of them (i.e., Monacolin K, 3 mg or 10 mg + Berberine 500 mg, Monacolin K + Fatty acids - w3).

Detailed diet information is reported in supplementary material, while the comprehensive diet composition is shown in Supplementary Table S1.

\subsection{Statistical Analysis}

Statistical analyses were performed using SPSS Statistics 25, Release Version 25.0; SPSS, Inc., 2017, Chicago, IL, USA (www.spss.com). Detailed statistical analysis is reported in the supplementary material.

\section{Results}

\subsection{Baseline Population Characteristics}

The flow chart in Figure 1 showed that patients on statin therapies returned to the follow-up visit more frequently than patients who received the diet alone $(1120,75.6 \%$ vs. $487,49.2 \%$, respectively) at Person' chi square test $(p<0.0001)$.

The clinical characteristics of all 487 subjects on the diet treatment with or without NUT supplementation, 201 men and 286 women, included in the main analysis were reported in Table 1. Most of the patients were natives of the North-West region of Italy, while only three patients were born in the Middle East (0.6\%) and four subjects in South America (0.8\%). All the subjects included lived in the Liguria region. The median follow-up period was 4 months (IQR 3-10 months) in patients treated with diet alone and five months (IQR 3-11 months) in subjects supplemented with NUT. No statistically significant differences have been observed among patients divided in the two different lifestyle modification approaches (i.e., Diet alone and diet plus NUT).

The differences between male and female gender in age, anthropometric measures, and smoking and alcohol consumption have been analyzed: men were younger than females, and males' weight and BMI were higher and tobacco and alcohol consumption (>10 g/day) was more frequent than in women (comprehensive characteristics have been reported in supplementary Table S2).

The baseline lipid profile of 487 subjects and the differences analyzed within patients divided according to gender, age, BMI, smoking habit, alcohol consumption, and treatment with or without NUT were reported in Supplementary Table S3. Females had a higher level of TC and HDL-C and a lower level of TG than males. HDL-C was significantly lower in younger subjects (age $<45$ years) than older ones. In overweight patients HDL-C and TG were respectively lower and higher than normal weight patients. No significant differences were observed in subgroups for LDL-C. Cross-sectional analyses were also performed of the relationships between the lipid profile and other variables included in the baseline study (Supplementary Table S4). The presence of lower TC and HDL-C and higher TG were independently correlated with male gender. Furthermore, higher HDL-C was independently correlated with normal weight patients while TG was independently correlated with overweight patients (Supplementary Table S4). 
Table 1. Baseline characteristics of 487 dyslipidemic non-statin treated patients divided in diet alone and diet plus NUT groups.

\begin{tabular}{|c|c|c|}
\hline Variable & Diet Alone & Diet + Nut \\
\hline Sex [F/M: n; \%] & $117(56.5 \%) / 90(43.5 \%)$ & $169(60.4 \%) / 111(39.6 \%)$ \\
\hline $\begin{array}{c}\text { Age } \\
\text { [years: mean } \pm \text { SD; median; IQ range] } \\
{[<45 \text { years } / \geq 45 \text { years: } n, \%]}\end{array}$ & $\begin{array}{c}50 \pm 15 ; 51(40,60) \\
78(37.7 \%) / 129(62.3 \%)\end{array}$ & $\begin{array}{c}52 \pm 13 ; 52(43,61) \\
88(31.4 \%) / 192(68.6 \%)\end{array}$ \\
\hline $\begin{array}{c}\text { Weight } \\
\text { [kg: mean } \pm \text { SD; median; IQ range] }\end{array}$ & $71.0 \pm 15.5 ; 69.0(60.0,81.0)$ & $69.2 \pm 14.3 ; 69.7(60.0,77.0)$ \\
\hline $\begin{array}{c}\text { BMI } \\
{\left[\mathrm{kg} / \mathrm{m}^{2}: \text { mean } \pm S D ; \text { median; IQ range }\right]} \\
{\left[<25 \mathrm{~kg} / \mathrm{m}^{2} / \geq 25 \mathrm{~kg} / \mathrm{m}^{2}\right]}\end{array}$ & $\begin{array}{l}25.2 \pm 4.3 ; 24.7(22.1,28.0) \\
\quad 110(53.1 \%) / 97(46.9 \%)\end{array}$ & $\begin{array}{c}24.8 \pm 3.8 ; 24.3(22.5,26.8) \\
166(59.3 \%) / 114(40.7 \%)\end{array}$ \\
\hline $\begin{array}{c}\mathrm{SBP} \\
{[\mathrm{mm} / \mathrm{Hg}: \text { mean } \pm \text { SD; median; IQ range] }}\end{array}$ & $126 \pm 2 ; 126(125,128)$ & $127 \pm 2 ; 127(125,128)$ \\
\hline $\begin{array}{c}\mathrm{DBP} \\
{[\mathrm{mm} / \mathrm{Hg}: \text { mean } \pm \text { SD; median; IQ range] }}\end{array}$ & $85 \pm 3 ; 85(83,87)$ & $85 \pm 3 ; 85(83,87)$ \\
\hline $\begin{array}{c}\text { Risk SCORE } \\
\text { [\%: mean } \pm \text { SD; median; IQ range] } \\
\text { Low-Risk: }<1 \%[\mathrm{n} ; \%] \\
\text { Moderate-Risk: } \geq 1 \% \text { and }<5 \% \text { [n; \%] } \\
\text { High-Risk: } \geq 5 \% \text { and }<10 \%[\mathrm{n} ; \%] \\
\text { Very-High-Risk: } \geq 10 \%[\mathrm{n} ; \%]\end{array}$ & $\begin{array}{c}2.1 \pm 3.0 ; 0.9(0.3,2.9) \\
117(56.5 \%) \\
64(30.9 \%) \\
22(10.6 \%) \\
4(1.9 \%)\end{array}$ & $\begin{array}{c}2.0 \pm 2.9 ; 0.9(0.3,2.5) \\
145(51.8 \%) \\
106(37.9 \%) \\
23(8.2 \%) \\
6(2.1 \%)\end{array}$ \\
\hline $\begin{array}{l}\text { Smoking habits } \\
\text { [Never + Past/Current: n; \%] }\end{array}$ & $155(74.9 \%) / 52(25.1 \%)$ & $218(77.9 \%) / 62(22.1 \%)$ \\
\hline $\begin{array}{l}\text { Alcohol intake } \\
\text { [No + Moderate/Excessive: n; \%] }\end{array}$ & $134(65.0 \%) / 72(35.0 \%)$ & $208(74.8 \%) / 70(25.2 \%)$ \\
\hline
\end{tabular}

Abbreviations: $\mathrm{M}=$ male, $\mathrm{F}=$ female, $\mathrm{y}=$ years, $\mathrm{BMI}=$ body mass index, $\mathrm{SBP}=$ systolic blood pressure, $\mathrm{DBP}=$ diastolic blood pressure, NUT $=$ lipid lowering nutraceutical.

\subsection{Effect of Diet Alone Treatment}

Between the baseline and follow-up, the median values of TC, LDL-C, and TG decreased by $-7.7 \%,-10.1 \%$, and $-16.7 \%$, respectively, in the 207 patients treated with diet alone. No significant variation was observed in the HDL-C value. A similar improvement in the lipid profile was observed in all analyzed subgroups of patients, however females were characterized by a larger reduction of LDL-C than males. Conversely, triglycerides decreased more in men than in women and in overweight than in normal weight patients (Table 2). A multivariate analysis (see statistical analysis for detailed formal description) showed a positive linear correlation between higher reduction in LDL-C and female gender and, on the other hand, a higher reduction in TG and male gender (Supplementary Table S5). 
Table 2. Effect of diet alone on lipid profile overall and in specific groups of patients.

\begin{tabular}{|c|c|c|c|c|c|c|c|c|c|c|c|c|}
\hline & \multicolumn{3}{|c|}{ TC } & \multicolumn{3}{|c|}{ HDL-C } & \multicolumn{3}{|c|}{ LDL-C } & \multicolumn{3}{|c|}{ TG } \\
\hline & $\begin{array}{l}\text { Baseline } \\
\text { (Median, } \\
\text { IQR) }\end{array}$ & $\begin{array}{l}\text { Follow-Up } \\
\text { (Median, } \\
\text { IQR) }\end{array}$ & $\begin{array}{c}\text { Variation }+ \\
\text { (Median, IQR, } \\
\%, p \text {-Value) }\end{array}$ & $\begin{array}{l}\text { Baseline } \\
\text { (Median, } \\
\text { IQR) }\end{array}$ & $\begin{array}{l}\text { Follow-Up } \\
\text { (Median, } \\
\text { IQR) }\end{array}$ & $\begin{array}{c}\text { Variation }+ \\
\text { (Median, IQR, } \\
\%, p \text {-Value) }\end{array}$ & $\begin{array}{l}\text { Baseline } \\
\text { (Median, } \\
\text { IQR) }\end{array}$ & $\begin{array}{l}\text { Follow-Up } \\
\text { (Median, } \\
\text { IQR) }\end{array}$ & $\begin{array}{c}\text { Variation }+ \\
\text { (Median, IQR, } \\
\%, p \text {-Value) }\end{array}$ & $\begin{array}{l}\text { Baseline } \\
\text { (Median, } \\
\text { IQR) }\end{array}$ & $\begin{array}{l}\text { Follow-Up } \\
\text { (Median, } \\
\text { IQR) }\end{array}$ & $\begin{array}{c}\text { Variation }+ \\
\text { (Median, IQR, } \\
\%, p \text {-Value) }\end{array}$ \\
\hline $\begin{array}{l}\text { All patients } \\
\text { (n:207) }\end{array}$ & $\begin{array}{c}259 \\
(241,285)\end{array}$ & $\begin{array}{c}237 \\
(205,260)\end{array}$ & $\begin{array}{c}-19(-46,-6) \\
-7,7 \%, p<0.001\end{array}$ & $\begin{array}{c}54 \\
(42,66)\end{array}$ & $\begin{array}{c}53 \\
(42,66)\end{array}$ & $\begin{array}{c}0(-6,5) \\
0.0 \%, \mathrm{NS}\end{array}$ & $\begin{array}{c}176 \\
(154,194)\end{array}$ & $\begin{array}{c}155 \\
(125,177)\end{array}$ & $\begin{array}{c}-18(-40,-1) \\
-10.1 \%, p<0.001\end{array}$ & $\begin{array}{c}142 \\
(96,232)\end{array}$ & $\begin{array}{c}120 \\
(82,173)\end{array}$ & $\begin{array}{c}-20(-68,7) \\
-16,7 \%, p<0.001\end{array}$ \\
\hline \multicolumn{13}{|l|}{ Sex* } \\
\hline $\begin{array}{c}\mathrm{F}(\mathrm{n}: 117, \\
56.5 \%)\end{array}$ & $\begin{array}{c}264 \\
(250,287)\end{array}$ & $\begin{array}{c}241 \\
(216,261)\end{array}$ & $\begin{array}{c}-22(-54,-10) \\
-8.4 \%, p<0.001\end{array}$ & $\begin{array}{c}61 \\
(50,73)\end{array}$ & $\begin{array}{c}62 \\
(48,73)\end{array}$ & $\begin{array}{c}1(-6,5) \\
0.7 \%, \mathrm{NS}\end{array}$ & $\begin{array}{c}178 \\
(159,192)\end{array}$ & $\begin{array}{c}157 \\
(128,176)\end{array}$ & $\begin{array}{c}-20(-45,-7) \\
-11.4 \%, p<0.001\end{array}$ & $\begin{array}{c}110 \\
(87,168)\end{array}$ & $\begin{array}{c}100 \\
(76,163)\end{array}$ & $\begin{array}{c}-13(-44,9) \\
-12.2 \%, p<0.001\end{array}$ \\
\hline $\begin{array}{c}\text { M (n: } 90 \\
43.5 \%)\end{array}$ & $\begin{array}{c}250 \\
(222,275)\end{array}$ & $\begin{array}{c}224 \\
(203,256)\end{array}$ & $\begin{array}{c}-15(-39,2) \\
-6.6 \%, p<0.001\end{array}$ & $\begin{array}{c}47 \\
(37,56)\end{array}$ & $\begin{array}{c}45 \\
(39,54)\end{array}$ & $\begin{array}{c}0(-6,5) \\
0.0 \% \text {, NS }\end{array}$ & $\begin{array}{c}169 \\
(137,196)\end{array}$ & $\begin{array}{c}153 \\
(122,178)\end{array}$ & $\begin{array}{c}-8(-30,8) \\
-5.6 \%, p=0.001\end{array}$ & $\begin{array}{c}197 \\
(130,315)\end{array}$ & $\begin{array}{c}136 \\
(105,189)\end{array}$ & $\begin{array}{c}-45(-150,-2) \\
-27.2 \%, p<0.001\end{array}$ \\
\hline \multicolumn{13}{|l|}{ Age * } \\
\hline $\begin{array}{c}<45 \text { years } \\
\text { (n: } 78,129 \%)\end{array}$ & $\begin{array}{c}256 \\
(222,284)\end{array}$ & $\begin{array}{c}228 \\
(200,256)\end{array}$ & $\begin{array}{c}-20(-45,-4) \\
-7.7 \%, p<0.001\end{array}$ & $\begin{array}{c}53 \\
(41,63)\end{array}$ & $\begin{array}{c}52 \\
(41,63)\end{array}$ & $\begin{array}{c}0(-7,5) \\
0.0 \%, \text { NS }\end{array}$ & $\begin{array}{c}170 \\
(152,192)\end{array}$ & $\begin{array}{c}151 \\
(117,174)\end{array}$ & $\begin{array}{c}-17(-41,3) \\
-9.9 \%, p<0.001\end{array}$ & $\begin{array}{c}154 \\
(101,229)\end{array}$ & $\begin{array}{c}124 \\
(80,172)\end{array}$ & $\begin{array}{c}-20(-73,8) \\
-14.9 \%, p<0.001\end{array}$ \\
\hline $\begin{array}{c}\geq 45 \text { years } \\
\text { (n: } 129,62.3 \%)\end{array}$ & $\begin{array}{c}261 \\
(246,287)\end{array}$ & $\begin{array}{c}240 \\
(212,261)\end{array}$ & $\begin{array}{c}-19(-49,-8) \\
-7.7 \%, p<0.001\end{array}$ & $\begin{array}{c}54 \\
(43,67)\end{array}$ & $\begin{array}{c}55 \\
(45,70)\end{array}$ & $\begin{array}{c}1(-6,5) \\
1.6 \% \text {, NS }\end{array}$ & $\begin{array}{c}177 \\
(158,196)\end{array}$ & $\begin{array}{c}158 \\
(130,178)\end{array}$ & $\begin{array}{c}-18(-40,-4) \\
-10.1 \%, p<0.001\end{array}$ & $\begin{array}{c}136 \\
(96,235)\end{array}$ & $\begin{array}{c}120 \\
(82,174)\end{array}$ & $\begin{array}{c}-21(-67,2) \\
-18.2 \%, p<0.001\end{array}$ \\
\hline \multicolumn{13}{|l|}{ BMI } \\
\hline $\begin{array}{c}<25 \mathrm{~kg} / \mathrm{m}^{2} \\
(\mathrm{n}: 110,53.1 \%)\end{array}$ & $\begin{array}{c}257 \\
(242,281)\end{array}$ & $\begin{array}{c}236 \\
(205,256)\end{array}$ & $\begin{array}{c}-20(-49,-6) \\
-7.5 \%, p<0.001\end{array}$ & $\begin{array}{c}59 \\
(49,70)\end{array}$ & $\begin{array}{c}59 \\
(47,68)\end{array}$ & $\begin{array}{c}0(-7,5) \\
0.0 \% \text {, NS }\end{array}$ & $\begin{array}{c}174 \\
(155,190)\end{array}$ & $\begin{array}{c}152 \\
(122,175)\end{array}$ & $\begin{array}{c}-19(-40,-3) \\
-11.1 \%, p<0.001\end{array}$ & $\begin{array}{c}117 \\
(82,180)\end{array}$ & $\begin{array}{c}101 \\
(77,154)\end{array}$ & $\begin{array}{c}-11(-47,11) \\
-11.1 \%, p<0.001\end{array}$ \\
\hline $\begin{array}{c}\geq 25 \mathrm{~kg} / \mathrm{m}^{2} \\
(\mathrm{n}: 97,46.9 \%)\end{array}$ & $\begin{array}{c}261 \\
(238,288)\end{array}$ & $\begin{array}{c}239 \\
(206,263)\end{array}$ & $\begin{array}{c}-17(-45,-6) \\
-8.1 \%, p<0.001\end{array}$ & $\begin{array}{c}48 \\
(40,60)\end{array}$ & $\begin{array}{c}47 \\
(40,63)\end{array}$ & $\begin{array}{c}1(-5,6) \\
2.2 \% \text {, NS }\end{array}$ & $\begin{array}{c}179 \\
(154,198)\end{array}$ & $\begin{array}{c}158 \\
(130,179)\end{array}$ & $\begin{array}{c}-16(-40,1) \\
-9.1 \%, p<0.001\end{array}$ & $\begin{array}{c}196 \\
(120,315)\end{array}$ & $\begin{array}{c}137 \\
(99,209)\end{array}$ & $\begin{array}{c}-40(-121,-7) \\
-23.5 \%, p<0.001\end{array}$ \\
\hline \multicolumn{13}{|l|}{$\begin{array}{l}\text { Smoking } \\
\text { habits * }\end{array}$} \\
\hline $\begin{array}{c}\text { Never/Past } \\
\text { (n: } 155,74.9 \%)\end{array}$ & $\begin{array}{c}262 \\
(241,287)\end{array}$ & $\begin{array}{c}240 \\
(205,261)\end{array}$ & $\begin{array}{c}-19(-44,-6) \\
-7.5 \%, p<0.001\end{array}$ & $\begin{array}{c}54 \\
(44,66)\end{array}$ & $\begin{array}{c}54 \\
(42,67)\end{array}$ & $\begin{array}{c}0(-6,5) \\
0.0 \%, \text { NS }\end{array}$ & $\begin{array}{c}177 \\
(155,197)\end{array}$ & $\begin{array}{c}156 \\
(127,179)\end{array}$ & $\begin{array}{c}-17(-38,-1) \\
-9.9 \%, p<0.001\end{array}$ & $\begin{array}{c}138 \\
(94,228)\end{array}$ & $\begin{array}{c}119 \\
(81,168)\end{array}$ & $\begin{array}{c}-18(-63,8) \\
-14.5 \%, p<0.001\end{array}$ \\
\hline $\begin{array}{c}\text { Current (n: 52, } \\
25.1 \%)\end{array}$ & $\begin{array}{c}257 \\
(237,277)\end{array}$ & $\begin{array}{c}227 \\
(206,251)\end{array}$ & $\begin{array}{c}-19(-52,-7) \\
-8.2 \%, p<0.001\end{array}$ & $\begin{array}{c}53 \\
(40,65)\end{array}$ & $\begin{array}{c}52 \\
(44,64)\end{array}$ & $\begin{array}{c}2 \\
(-6,10) 4.7 \%, \mathrm{NS}\end{array}$ & $\begin{array}{c}171 \\
(152,187)\end{array}$ & $\begin{array}{c}153 \\
(121,172)\end{array}$ & $\begin{array}{c}-18(-45,1) \\
-11.0 \%, p<0.001\end{array}$ & $\begin{array}{c}161 \\
(102,320)\end{array}$ & $\begin{array}{c}123 \\
(88,185)\end{array}$ & $\begin{array}{c}-37(-147,-2) \\
-26.3 \%, p<0.001\end{array}$ \\
\hline \multicolumn{13}{|l|}{$\begin{array}{c}\text { Alcohol } \\
\text { consumption } \\
*\end{array}$} \\
\hline $\begin{array}{c}\text { Absent/moderate } \\
(135,64.3 \%)\end{array}$ & $\begin{array}{c}262 \\
(237,288)\end{array}$ & $\begin{array}{c}234 \\
(205,260)\end{array}$ & $\begin{array}{c}-22(-53,-6) \\
-8.4 \%, p<0.001\end{array}$ & $\begin{array}{c}53 \\
(41,66)\end{array}$ & $\begin{array}{c}54 \\
(42,67)\end{array}$ & $\begin{array}{c}1(-4,6) \\
2.6 \%, \text { NS }\end{array}$ & $\begin{array}{c}179 \\
(155,194)\end{array}$ & $\begin{array}{c}154 \\
(121,176)\end{array}$ & $\begin{array}{c}-20(-45,-2) \\
-11.4 \%, p<0.001\end{array}$ & $\begin{array}{c}143 \\
(96,235)\end{array}$ & $\begin{array}{c}115 \\
(81,168)\end{array}$ & $\begin{array}{c}-20(-68,8) \\
-16.2 \%, p<0.001\end{array}$ \\
\hline $\begin{array}{c}\text { Elevate (72, } \\
35.7 \%)\end{array}$ & $\begin{array}{c}257 \\
(242,278)\end{array}$ & $\begin{array}{c}237 \\
(208,262)\end{array}$ & $\begin{array}{c}-16(-40,-4) \\
-6.0 \%, p<0.001\end{array}$ & $\begin{array}{c}54 \\
(46,65)\end{array}$ & $\begin{array}{c}52 \\
(43,64)\end{array}$ & $\begin{array}{l}-1(-7,4) \\
-2.1 \%, \text { NS }\end{array}$ & $\begin{array}{c}170 \\
(152,194)\end{array}$ & $\begin{array}{c}157 \\
(133,178)\end{array}$ & $\begin{array}{c}-16(-29,8) \\
-8.4 \%, p=0.01\end{array}$ & $\begin{array}{c}134 \\
(96,229)\end{array}$ & $\begin{array}{c}132 \\
(82,177)\end{array}$ & $\begin{array}{c}-19(-67,6) \\
-18.2 \%, p<0.001\end{array}$ \\
\hline
\end{tabular}




\subsection{Effect of Lipid Lowering Nutraceuticals}

Overall, 280 subjects underwent lipid lowering treatment with diet plus NUT. General characteristics and lipid parameters have been reported in supplementary Table S6. However, formal analysis considered only patients in three therapy groups: (1) 29 subjects with MonK $(3 \mathrm{mg} / \mathrm{day}$ or $10 \mathrm{mg} /$ day), (2) 167 subjects with Monk ( $3 \mathrm{mg} /$ day or $10 \mathrm{mg} /$ day) plus BBR (500 mg/day), and (3) 36 patients with PUFA-W3 ( $3 \mathrm{~g} /$ day). Supplements used in less than 11 subjects have been excluded as well as products with uncertain component titration (i.e., pharmacy galenic formulas). Variation in lipid parameters between baseline and follow-up within the three groups have been reported in Table 3, while medians of percentage variations have been shown in Figure 2. Moreover, the differences in median values have been calculated within NUT groups and diet alone (Table 3). The most effective NUTs group in reducing LDL-C was MonK plus BBR, which was also more effective than PUFA Omega $3(p<0.0001)$ and MonK alone $(p=0.294, N S)$. TG reduction was greater in patients treated with PUFA Omega 3 compared to diet alone $(p<0.049)$, MonK $(p=0.058$, NS) and MonK plus $\operatorname{BBR}(p<0.0001)$.

Table 3. Effect of different groups of NUTs on lipid profile and comparison with diet alone by the difference in the median.

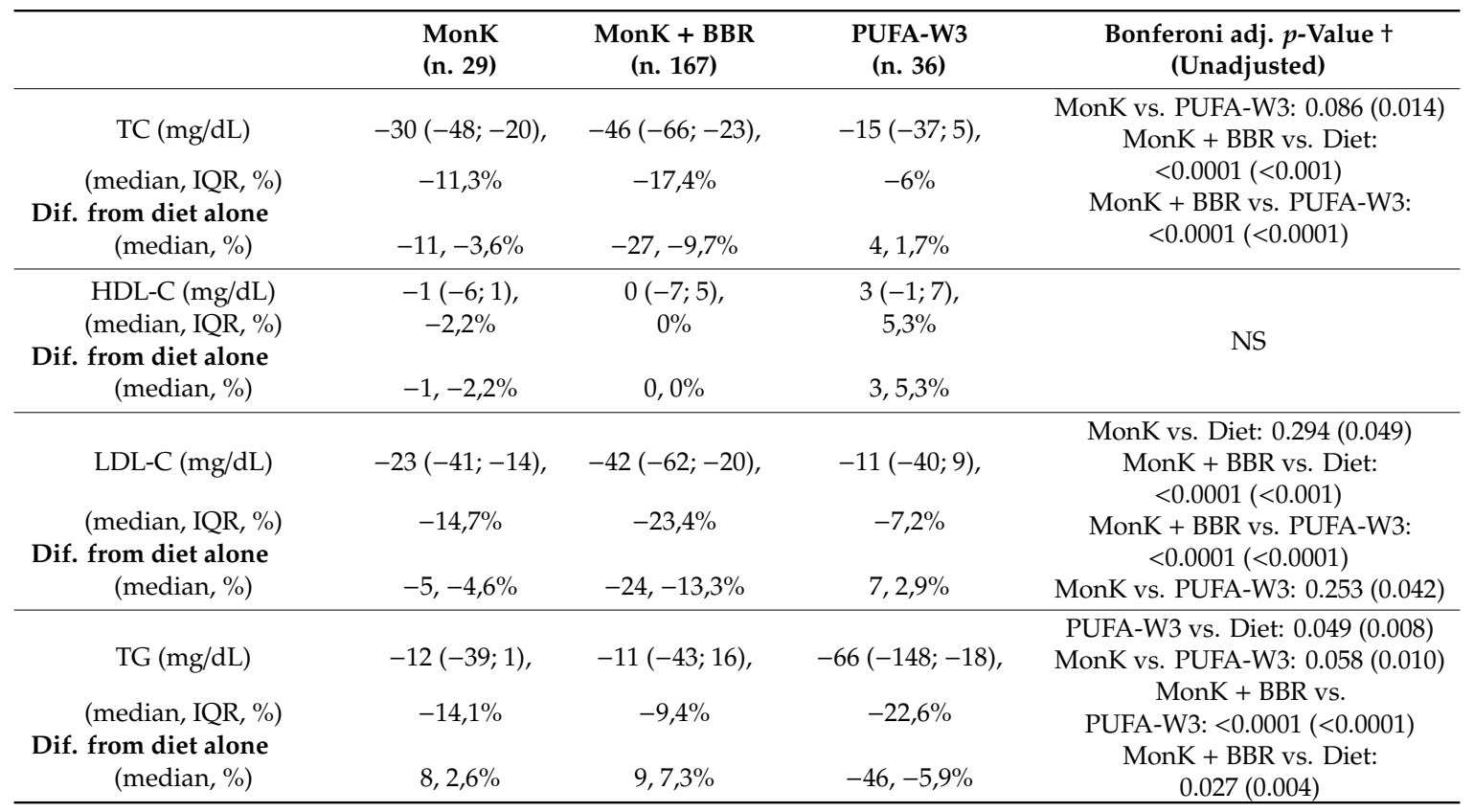

Data are variation of lipid profile variable and are presented as median, IQR and percentage of variation. Difference in median from diet alone are also shown. Abbreviations: MonK $=$ monacolin $(10 \mathrm{mg} /$ day $)$, MonK $+\mathrm{BBR}=$ monacolin (3-10 mg/day) plus berberine ( $500 \mathrm{mg} /$ day), PUFA-W3 $=\Omega 3-$ polyunsaturated fatty acid. + Indipendent samples Kruskal-Wallis tests. Significance values have been adjusted by the Bonferroni correction for multiple tests. Unadjusted $p$-values have been also reported. 


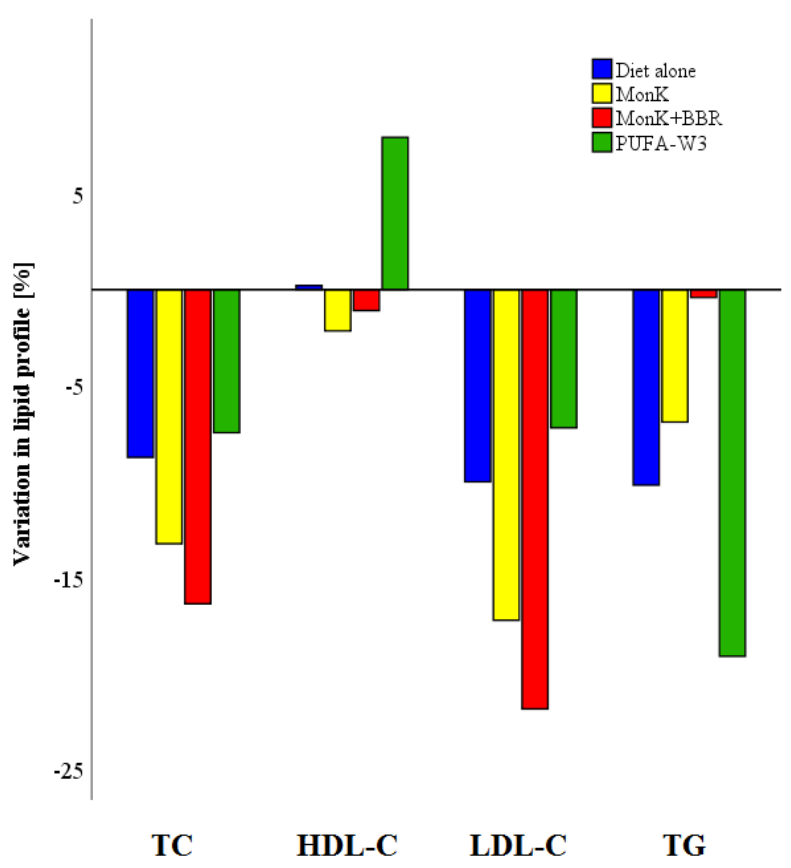

Figure 2. Percentage variation of lipid parameters in different NUTs groups.

\subsection{Lipid Lowering Intervention and Therapeutic Targets}

Patients at baseline visit were stratified according to their ASCVD risk through SCORE algorithm or diagnosing a pathological condition, such as familial hypercholesterolemia, familial combined hyperlipidemia or other ASCVD risk related conditions [13,14]. Table 4 reports the different success of our non-statin treatment (i.e., diet and diet plus NUT) in reaching 2016 and 2019 ESC/EAS LDL-C targets. At follow-up, the goals of the 2016 ESC/EAS guidelines were achieved in 40/262 (15.3\%) and 23/170 (13.5\%) respectively in low and moderate ASCVD risk (recommended LDL-C $<115 \mathrm{mg} / \mathrm{dL}$ ). However, 40/262 (15.3\%) and 11/170 (6.5\%) patients reached the 2019 ESC/EAS recommended LDL-C values in low (LDL-C $\leq 115 \mathrm{mg} / \mathrm{dL}$ ) and moderate (LDL-C $<100 \mathrm{mg} / \mathrm{dL}$ ) ASCVD risks. Only 3 out of 45 high-risk patients reached the 2016 ESC/EAS recommended LDL-C values, and none of the very-high risk subjects (n. 10) reached the values. Conversely, no patients in high or very-high ASCVD risk classes reached the 2019 ESC/EAS recommended LDL-C goals.

Table 4. Patients reaching and not reaching 2016 and 2019 ESC/EAS LDL-C recommended target in the different risk classes.

\begin{tabular}{|c|c|c|c|c|c|}
\hline \multicolumn{2}{|c|}{ ASCVD Risk } & $\begin{array}{l}\text { Low } \\
(<1 \%)\end{array}$ & $\begin{array}{l}\text { Moderate } \\
(\geq 1 \% ;<5 \%)\end{array}$ & $\begin{array}{c}\text { High } \\
(\geq 5 \% ;<10 \%)\end{array}$ & $\begin{array}{l}\text { Very High } \\
(\geq 10 \%)\end{array}$ \\
\hline \multicolumn{2}{|c|}{2016 ESC/EAS LDL-C targets } & $<115 \mathrm{mg} / \mathrm{dL}$ & $<115 \mathrm{mg} / \mathrm{dL}$ & $<100 \mathrm{mg} / \mathrm{dL}$ & $<70 \mathrm{mg} / \mathrm{dL}$ \\
\hline & & & Baseline & & \\
\hline \multirow{3}{*}{$\begin{array}{l}\text { Reaching } 2016 \\
\text { ESC/EAS Guidelines }\end{array}$} & n (\%) & $8(3.1 \%)$ & $7(4.1 \%)$ & $0(0 \%)$ & $0(0 \%)$ \\
\hline & $\mathrm{F} / \mathrm{M}$ & $3 / 5$ & $3 / 4$ & NA & NA \\
\hline & Age (years) & $40(33-45)$ & $64(57-68)$ & NA & NA \\
\hline \multirow{4}{*}{$\begin{array}{l}\text { Not reaching } 2016 \\
\text { ESC/EAS Guidelines }\end{array}$} & $\mathrm{n}(\%)$ & $254(96.9 \%)$ & $163(95.9 \%)$ & $45(100 \%)$ & $10(100 \%)$ \\
\hline & $\mathrm{F} / \mathrm{M}$ & $150 / 104$ & $104 / 59$ & $22 / 23$ & $4 / 6$ \\
\hline & Age (years) & $43(36-50)$ & $59(55-65)$ & $68(64-74)$ & $74(73-77)$ \\
\hline & & & Follow-up & & \\
\hline
\end{tabular}


Table 4. Cont.

\begin{tabular}{|c|c|c|c|c|c|}
\hline \multicolumn{2}{|c|}{ ASCVD Risk } & $\begin{array}{l}\text { Low } \\
(<1 \%)\end{array}$ & $\begin{array}{c}\text { Moderate } \\
(\geq 1 \% ;<5 \%)\end{array}$ & $\begin{array}{c}\text { High } \\
(\geq 5 \% ;<10 \%)\end{array}$ & $\begin{array}{c}\text { Very High } \\
(\geq 10 \%)\end{array}$ \\
\hline \multirow{3}{*}{$\begin{array}{c}\text { Reaching } 2016 \\
\text { ESC/EAS Guidelines }\end{array}$} & $\mathrm{n}(\%)$ & $40(15.3 \%)$ & $23(13.5 \%)$ & $3(6.7 \%)$ & $0(0 \%)$ \\
\hline & $\mathrm{F} / \mathrm{M}$ & $23 / 17$ & $16 / 7$ & $2 / 1$ & NA \\
\hline & Age (years) & $41(33-49)$ & $61(58-65)$ & $74(69-77)$ & NA \\
\hline \multirow{3}{*}{$\begin{array}{l}\text { Not reaching } 2016 \\
\text { ESC/EAS Guidelines }\end{array}$} & n (\%) & $222(84.7 \%)$ & 147 (86.5\%) & $42(93.3 \%)$ & $10(100 \%)$ \\
\hline & $\mathrm{F} / \mathrm{M}$ & $130(58.6 \%)$ & $91(61.9 \%)$ & $20(46.5 \%)$ & $4(40.0 \%)$ \\
\hline & Age (years) & $43(36-50)$ & $59(55-65)$ & $68(64-73)$ & $74(73-77)$ \\
\hline \multicolumn{2}{|c|}{2019 ESC/EAS LDL-C targets } & $<116 \mathrm{mg} / \mathrm{dL}$ & $<100 \mathrm{mg} / \mathrm{dL}$ & $<70 \mathrm{mg} / \mathrm{dL}$ & $<55 \mathrm{mg} / \mathrm{dL}$ \\
\hline & & & Baseline + & & \\
\hline \multirow{3}{*}{$\begin{array}{c}\text { Reaching } 2019 \\
\text { ESC/EAS Guidelines }\end{array}$} & $\mathrm{n}(\%)$ & $8(3.1 \%)$ & $1(0.6 \%)$ & $0(0 \%)$ & $0(0 \%)$ \\
\hline & $\mathrm{F} / \mathrm{M}$ & $3 / 5$ & $1 / 0$ & NA & NA \\
\hline & Age (years) & $40(33-45)$ & 70 & NA & NA \\
\hline \multirow{4}{*}{$\begin{array}{l}\text { Not reaching } 2019 \\
\text { ESC/EAS Guidelines }\end{array}$} & $\mathrm{n}(\%)$ & $254(96.9 \%)$ & $169(99.4 \%)$ & $45(100 \%)$ & $10(100 \%)$ \\
\hline & $\mathrm{F} / \mathrm{M}$ & 150/104 & $106 / 63$ & $22 / 23$ & $4 / 6$ \\
\hline & Age (years) & $43(36-50)$ & $59(55-65)$ & $68(64-74)$ & $74(73-77)$ \\
\hline & & & Follow-up & & \\
\hline \multirow{3}{*}{$\begin{array}{c}\text { Reaching } 2019 \\
\text { ESC/EAS Guidelines }\end{array}$} & $\mathrm{n}(\%)$ & $40(15.3 \%)$ & $11(6.5 \%)$ & $0(0 \%)$ & $0(0 \%)$ \\
\hline & $\mathrm{F} / \mathrm{M}$ & $23 / 17$ & $9 / 2$ & NA & NA \\
\hline & Age (years) & $41(33-49)$ & $62(58-65)$ & NA & NA \\
\hline \multirow{3}{*}{$\begin{array}{l}\text { Not reaching } 2019 \\
\text { ESC/EAS Guidelines }\end{array}$} & $\mathrm{n}(\%)$ & $222(84.7 \%)$ & $159(93.5 \%)$ & $45(100 \%)$ & $10(100 \%)$ \\
\hline & $\mathrm{F} / \mathrm{M}$ & $130 / 92$ & $98 / 61$ & $22 / 23$ & $4 / 6$ \\
\hline & Age (years) & $43(36-50)$ & $59(55-65)$ & $68(64-74)$ & $74(73-77)$ \\
\hline
\end{tabular}

Significant difference $(p<0.0001)$ at Pearson's chi-squared test vs. follow-up within patient's ASCVD risk distribution., + Significant difference $(p<0.0001)$ at Pearson's chi-squared test vs. follow-up within patient's ASCVD risk distribution.

On the other hand, lifestyle modification successfully increased the percentage of patients reaching LDL-C goals after the follow-up period (Table 4).

\section{Discussion}

The main purpose of this study was to evaluate the effect of diet treatment and NUTs on plasma lipid parameters in a real-world setting, taking into account possible influencing factors (i.e., gender, age, BMI, alcohol consumption, and smoking habits). The recommended lipid goals of the guidelines for the management of dyslipidemias have been used as a reference to estimate the clinical efficacy of administered interventions.

A preliminary result is the higher percentage of pharmacologically-treated patients (i.e., with statins, ezetimibe, etc.) who returned to the follow-up visit compared to patients who received only the lifestyle intervention. Some studies reported that the adherence to lifestyle intervention was unsatisfactory and significantly lower than in patients treated with drugs [32-34]. Thus, our results may be related to patients' altered behavior between diet and drugs and our study cases probably considered drug therapy more effective and important than lifestyle intervention. Thus, patients treated only with lifestyle intervention returned less frequently to the follow-up visit and may have considered lifestyle intervention as a second-rate therapy.

An important result of the study was the significant reduction of TC $(-19 \mathrm{mg} / \mathrm{dL},-7.7 \%)$, LDL-C $(-18 \mathrm{mg} / \mathrm{dL},-10.1 \%)$ and TG $(-20 \mathrm{mg} / \mathrm{dL},-16.7 \%)$ with the administration of a diet alone treatment. We observed an improvement of the lipid profile, which was consistent with the literature despite the lifestyle intervention having been conducted in an outpatient setting and the dietitian counseling lasting only $10 \mathrm{~min}$ on average. An important position paper of an International Lipid 
Expert Panel reported an LDL-C reduction between 5\% and 15\% with lifestyles interventions based on the Mediterranean diet [24]. Likewise, there are no unequivocal data on the reduction of TG that is reported between $5 \%$ and $20 \%$ [35], but with important differences among subjects.

Actually, some cross-sectional studies reported that the female gender is associated with higher HDL-C and lower TG levels [36,37] but female adherence to lifestyle modifications is higher than men [38]. The present data showed that the characteristics of the baseline lipid profile and the reduction of LDL-C in males and females agreed with the literature, except for TG levels, which decreased more in men. The differences in the basal lipid profile and in the dietary effects could be related to specific and not quantifiable traits of male gender (i.e., psychological, social, and cultural patterns) but also to a worse anthropometric and lifestyle features observed in our study cases. Men were found to have higher BMI and alcohol consumption and worse smoking habits than women. This phenotypic characteristic is consistent with literature: hypertriglyceridemia was related to high alcohol intake, obesity [39], and male gender [40], while low HDL-C levels were also associated with smoking habits [41], obesity, and male gender. On the other hand, the larger decrease in TG observed in men was probably due to the higher basal levels in comparison to women in which levels were substantially normal.

Subjects who received NUTs have been characterized by a greater reduction of lipid parameters than from diet alone. In particular, the LDL-C and TC reduction with MonK was $-14.7 \%$ and $-11.3 \%$, respectively, while other studies reported a percentage variation ranging from $-27.3 \%$ to $-12.5 \%$ and from $-15.5 \%$ to $-6.6 \%$ in LDL-C and TC, respectively [42-47]. Noteworthy, the combination of MonK and BBR was the most effective NUT in reducing LDL-C and TC $(-23.4 \%$ and $-17.4 \%$, respectively). This finding is consistent with literature $[24,48]$ and could be useful in patients with low ASCVD risk, even if the guidelines for the management of dyslipidemias highlighted the lack of high-quality randomized clinical trials. Furthermore, this type of NUTs as an addition to the diet could have various beneficial effects on the risk of atherosclerosis not only related to LDL-C reduction, as previously documented in some publications by other groups and ours $[49,50]$. On the other hand, the diet supplemented with PUFA-W3 was the most effective TG lowering treatment with a median percentage reduction of $-22.6 \%$ from baseline to follow-up. This outcome agreed with a recent metanalysis which showed the efficacy of $3 \mathrm{~g} /$ day of PUFA-W3 in reducing TG of about $14 \%$ [51].

Another relevant finding emerging from our study was the poor success in our patients with both lifestyle interventional strategies (i.e., diet alone and diet plus NUT) to reach the new LDL-C goals recommended by ESC/EAS guidelines. In fact, after the follow-up period, only 40 (15. 3\%) and 11 (6.5\%) subjects out of 262 and 170 in low and moderate ASCVD risk, respectively, were at ESC/EAS 2019 [14] targets and no patients in high and very-high risk classes reached the targets. The results obtained considering the 2016 targets were comparable with the reported analysis of the 2019 targets. Only a few more patients at moderate and high ACSVD risk achieved the less ambitious LDL-C targets of 2016 ESC/EAS guidelines (n. 23 with LDL-C $<115 \mathrm{mg} / \mathrm{dL}$ vs. $n$. 11 with LDL-C $<100 \mathrm{mg} / \mathrm{dL}$ in moderate risk class and n. 3 with LDL-C $<100 \mathrm{mg} / \mathrm{dL}$ vs. $n$. 0 with LDL-C $<70 \mathrm{mg} / \mathrm{dL}$ in high risk class, respectively).

All patients not reaching LDL-C goals should undergo administration of a pharmacological lipid-lowering drug such as statins. The latter is undoubtedly the best clinical strategy in patients at moderate to very-high ASCVD risk, except for contraindications. However, in our analysis, the patients at high and very-high ASCVD risk were not treated with drugs at baseline due to clinical history of statin intolerance or patients' decision, despite the guideline's recommendation. In low ASCVD risk subjects, different from the 2016 ESC/EAS guidelines which did not recommended pharmacological therapy if LDL-C $<190 \mathrm{mg} / \mathrm{dL}$, the $2019 \mathrm{ESC} / \mathrm{EAS}$ guidelines recommend also the use of drug therapy when lifestyle intervention was unsatisfactory in reducing LDL-C levels of at least $50 \%$ and less than $116 \mathrm{mg} / \mathrm{dL}$ (Class IIb and level of evidence A). However, the scientific literature is not conclusive on the need for statins in persons at low risk of ASCVD [52]. The reasons may be the high number of patients needed to treat to avoid nonfatal ASCVD (1:217 for myocardial infarction and 1:313 for stroke) [53], the risk of new onset type 2 diabetes mellitus [54] and the risk of statin-related side effects, such as 
musculoskeletal pain, elevation in creatine phosphokinase and hepatic dysfunctions [55]. Moreover, the Italian Ministry of Health authorized the refund of statins and other lipid-lowering drugs only for patients with ASCVD risk higher than 2\% [28]. Finally, the patients belonging to the low-risk class who did not reach LDL-C targets were mainly woman (n. 130/222, 58.6\%), non-smokers (n. 190/222, 85.6\%), normotensives (median SBP 126, IQR 124-127 and median DBP 84, IQR 81-86) and relatively younger patients (median 43, IQR 36-50 years). In our clinical experience, the low-risk patients with such described clinical characteristics are often resistant to accept pharmacological treatments. This observation is supported by literature which reported that the young age, the female gender and the absence of cardiovascular risk factors were associated with less adherence and prescription of statin therapy [56].

An important limitation of this study is represented by the relatively small sample size analyzed. This limit may have interfered with the possibility of carrying out more subgroup analyzes with greater statistical power. In light of this issue, we believe that new studies are necessary to confirm our results and we will consider the increasing of the patients' size as a priority in the near future. Another limitation of our study is the lack of the use of objective methods (i.e., food frequency questionnaire, food diary) to assess the compliance to the lifestyle intervention.

\section{Conclusions}

A lipid-lowering diet based on Mediterranean models improves lipid profiles in an outpatient real-world setting. The addition of NUTs can boost dietary efficacy, in particular MonK and its association with Berberine reduce LDL-C more than diet, while the larger improvement of triglyceridemia has been recorded with PUFA-W3 supplementation. However, all lifestyle modifications were mainly unsatisfactory with respect to the targets imposed by EAS/ESC guidelines, but the pharmacological treatment with statins in patients with low risk of ASCVD is still under discussion. Further studies will be needed to confirm our results and optimize the treatment of lipid risk factors, particularly in patients with low ASCVD risk.

Supplementary Materials: The following are available online at http://www.mdpi.com/2072-6643/12/7/2056/s1, Table S1: Composition of diets for overweight patients, Supplementary material 1.1: General advices on weekly food frequency in normal weight patients with primary hypercholesterolemia, Supplementary material 1.2: General advices on weekly food frequency with low carbohydrate intake in normal weight patients with hypertriglyceridemia or mixed hyperlipemia, Supplementary material 1.3: Diet with weekly food frequency and total energy intake of $1700 \mathrm{kcal} /$ day in overweight women, Supplementary material 1.4: Diet with weekly food frequency and total energy intake of $2100 \mathrm{kcal} /$ day in overweight men, Statistical analysis, Supplementary table S2.: General characteristics of patients according to intervention types and gender, Supplementary table S3: Baseline lipid profile in all patients and divided in subgroups according to sex, age, BMI, smoking habits, alcohol consumption and types of lifestyle intervention, Supplementary table S4: Multivariate analysis on baseline lipid profile in all 487 patients, Supplementary table S5: Multivariate analysis between baseline and follow-up of lipid profile in 207 patients on diet alone treatment, Supplementary table S6: General characteristics and lipid parameters of 280 patients on diet plus NUT treatment.

Author Contributions: Conceptualization, L.P.; Data curation, A.P., E.M., E.P. and S.P.; Formal analysis, A.P.; Investigation, A.P., E.F., A.L.C., E.M., E.P., S.P. and L.P.; Methodology, L.P.; Project administration, L.P.; Supervision, L.P.; Writing - original draft, A.P. and E.F.; Writing - review \& editing, A.L.C. and L.P. All authors have read and agreed to the published version of the manuscript.

Funding: This research received no external funding.

Conflicts of Interest: The authors declare no conflict of interest. 


\section{Abbreviations}

$\begin{array}{ll}\text { ASCVD } & \text { atherosclerotic cardiovascular disease } \\ \text { LDL-C } & \text { low-density lipoprotein cholesterol } \\ \text { TC } & \text { total cholesterol } \\ \text { HDL-C } & \text { high-density lipoprotein cholesterol } \\ \text { TG } & \text { triglycerides } \\ \text { PCSK9 } & \text { proprotein convertase subtilisin/kexin type 9 } \\ \text { BMI } & \text { body mass index } \\ \text { NUT } & \text { lipid lowering nutraceutical } \\ \text { MonK } & \text { Monacolin K } \\ \text { PS } & \text { Phytosterols } \\ \text { BBR } & \text { Berberine } \\ \text { PUFA-W3 } & \text { Fatty acids - } \omega 3 \\ \mathrm{n} & \text { number }\end{array}$

\section{References}

1. Yu, J.N.; Cunningham, J.A.; Thouin, S.R.; Gurvich, T.; Liu, D. Hyperlipidemia. Prim. Care 2000, 27, 541-587. [CrossRef]

2. Nelson, R.H. Hyperlipidemia as a Risk Factor for Cardiovascular Disease. Prim. Care 2013, 40, $195-211$. [CrossRef] [PubMed]

3. Roy, S. Atherosclerotic Cardiovascular Disease Risk and Evidence-based Management of Cholesterol. N Am. J. Med. Sci. 2014, 6, 191-198. [CrossRef] [PubMed]

4. Cabezas, M.C.; Burggraaf, B.; Klop, B. Dyslipidemias in clinical practice. Clin. Chim. Acta 2018, 487, 117-125. [CrossRef] [PubMed]

5. Scherer, J.; Singh, V.P.; Pitchumoni, C.S.; Yadav, D. Issues in hypertriglyceridemic pancreatitis: An update. J. Clin. Gastroenterol. 2014, 48, 195-203. [CrossRef]

6. Vega, G.L.; Grundy, S.M. Hypoalphalipoproteinemia (low high density lipoprotein) as a risk factor for coronary heart disease. Curr. Opin. Lipidol. 1996, 7, 209-216. [CrossRef]

7. Ziaeian, B.; Fonarow, G.C. Statins and the Prevention of Heart Disease. JAMA Cardiol. 2017, 2, 464. [CrossRef]

8. Sampson, U.K.; Fazio, S.; Linton, M.F. Residual cardiovascular risk despite optimal LDL cholesterol reduction with statins: The evidence, etiology, and therapeutic challenges. Curr. Atheroscler. Rep. 2012, 14, 1-10. [CrossRef]

9. Toth, P.P.; Catapano, A.; Tomassini, J.E.; Tershakovec, A.M. Update on the efficacy and safety of combination ezetimibe plus statin therapy. Clin. Lipidol. 2010, 5, 655-684. [CrossRef]

10. Morrone, D.; Weintraub, W.S.; Toth, P.P.; Hanson, M.E.; Lowe, R.S.; Lin, J.; Shah, A.K.; Tershakovec, A.M. Lipid-altering efficacy of ezetimibe plus statin and statin monotherapy and identification of factors associated with treatment response: A pooled analysis of over 21,000 subjects from 27 clinical trials. Atherosclerosis 2012, 223, 251-261. [CrossRef]

11. Pasta, A.; Cremonini, A.L.; Pisciotta, L.; Buscaglia, A.; Porto, I.; Barra, F.; Ferrero, S.; Brunelli, C.; Rosa, G.M. PCSK9 inhibitors for treating hypercholesterolemia. Expert Opin. Pharm. 2020, 21, 353-363. [CrossRef] [PubMed]

12. Remick, J.; Weintraub, H.; Setton, R.; Offenbacher, J.; Fisher, E.; Schwartzbard, A. Fibrate therapy: An update. Cardiol. Rev. 2008, 16, 129-141. [CrossRef] [PubMed]

13. Catapano, A.L.; Graham, I.; De Backer, G.; Wiklund, O.; Chapman, M.J.; Drexel, H.; Hoes, A.W.; Jennings, C.S.; Landmesser, U.; Pedersen, T.R.; et al. 2016 ESC/EAS Guidelines for the Management of Dyslipidaemias. Eur. Heart J. 2016, 37, 2999-3058. [CrossRef] [PubMed]

14. Mach, F.; Baigent, C.; Catapano, A.L.; Koskinas, K.C.; Casula, M.; Badimon, L.; Chapman, M.J.; De Backer, G.G.; Delgado, V.; Ference, B.A.; et al. 2019 ESC/EAS Guidelines for the management of dyslipidaemias: Lipid modification to reduce cardiovascular risk. Eur. Heart J. 2020, 41, 111-188. [CrossRef]

15. American Heart Association Nutrition Committee; Lichtenstein, A.H.; Appel, L.J.; Brands, M.; Carnethon, M.; Daniels, S.; Franch, H.A.; Franklin, B.; Kris-Etherton, P.; Harris, W.S. Diet and lifestyle recommendations 
revision 2006: A scientific statement from the American Heart Association Nutrition Committee. Circulation 2006, 114, 82-96. [CrossRef]

16. Trichopoulou, A.; Lagiou, P. Healthy traditional Mediterranean diet: An expression of culture, history, and lifestyle. Nutr. Rev. 1997, 55, 383-389. [CrossRef]

17. Brinton, E.A. Management of Hypertriglyceridemia for Prevention of Atherosclerotic Cardiovascular Disease. Endocrinol. Metab. Clin. N. Am. 2016, 45, 185-204. [CrossRef]

18. Obarzanek, E.; Sacks, F.M.; Vollmer, W.M.; Bray, G.A.; Miller, E.R.; Lin, P.H.; Karanja, N.M.; Most-Windhauser, M.M.; Moore, T.J.; Swain, J.F.; et al. Effects on blood lipids of a blood pressure-lowering diet: The Dietary Approaches to Stop Hypertension (DASH) Trial. Am. J. Clin. Nutr. 2001, 74, 80-89. [CrossRef]

19. Appel, L.J.; Sacks, F.M.; Carey, V.J.; Obarzanek, E.; Swain, J.F.; Miller, E.R.; Conlin, P.R.; Erlinger, T.P.; Rosner, B.A.; Laranjo, N.M.; et al. Effects of protein, monounsaturated fat, and carbohydrate intake on blood pressure and serum lipids: Results of the OmniHeart randomized trial. JAMA 2005, 294, 2455-2464. [CrossRef]

20. Ginsberg, H.N.; Kris-Etherton, P.; Dennis, B.; Elmer, P.J.; Ershow, A.; Lefevre, M.; Pearson, T.; Roheim, P.; Ramakrishnan, R.; Reed, R.; et al. Effects of reducing dietary saturated fatty acids on plasma lipids and lipoproteins in healthy subjects: The DELTA Study, protocol 1. Arter. Thromb. Vasc. Biol. 1998, 18, 441-449. [CrossRef]

21. Schaefer, E.J.; Lamon-Fava, S.; Ausman, L.M.; Ordovas, J.M.; Clevidence, B.A.; Judd, J.T.; Goldin, B.R.; Woods, M.; Gorbach, S.; Lichtenstein, A.H. Individual variability in lipoprotein cholesterol response to National Cholesterol Education Program Step 2 diets. Am. J. Clin. Nutr. 1997, 65, 823-830. [CrossRef]

22. Banach, M.; Patti, A.M.; Giglio, R.V.; Cicero, A.F.G.; Atanasov, A.G.; Bajraktari, G.; Bruckert, E.; Descamps, O.; Djuric, D.M.; Ezhov, M.; et al. The Role of Nutraceuticals in Statin Intolerant Patients. J. Am. Coll. Cardiol. 2018, 72, 96-118. [CrossRef]

23. Patti, A.M.; Toth, P.P.; Giglio, R.V.; Banach, M.; Noto, M.; Nikolic, D.; Montalto, G.; Rizzo, M. Nutraceuticals as an Important Part of Combination Therapy in Dyslipidaemia. Curr. Pharm. Des. 2017, 23, 2496-2503. [CrossRef]

24. Cicero, A.F.G.; Colletti, A.; Bajraktari, G.; Descamps, O.; Djuric, D.M.; Ezhov, M.; Fras, Z.; Katsiki, N.; Langlois, M.; Latkovskis, G.; et al. Lipid-lowering nutraceuticals in clinical practice: Position paper from an International Lipid Expert Panel. Nutr. Rev. 2017, 75, 731-767. [CrossRef]

25. Backes, J.; Anzalone, D.; Hilleman, D.; Catini, J. The clinical relevance of omega-3 fatty acids in the management of hypertriglyceridemia. Lipids Health Dis. 2016, 15, 118. [CrossRef]

26. Arca, M.; Borghi, C.; Pontremoli, R.; De Ferrari, G.M.; Colivicchi, F.; Desideri, G.; Temporelli, P.L. Hypertriglyceridemia and omega-3 fatty acids: Their often overlooked role in cardiovascular disease prevention. Nutr. Metab. Cardiovasc Dis. 2018, 28, 197-205. [CrossRef]

27. McKenney, J.M.; Sica, D. Role of prescription omega-3 fatty acids in the treatment of hypertriglyceridemia. Pharmacotherapy 2007, 27, 715-728. [CrossRef]

28. Nota 13|Agenzia Italiana del Farmaco. Available online: https:/aifa.gov.it/nota-13 (accessed on 15 March 2020).

29. Conroy, R.M.; Pyörälä, K.; Fitzgerald, A.P.; Sans, S.; Menotti, A.; De Backer, G.; De Bacquer, D.; Ducimetière, P.; Jousilahti, P.; Keil, U.; et al. Estimation of ten-year risk of fatal cardiovascular disease in Europe: The SCORE project. Eur. Heart J. 2003, 24, 987-1003. [CrossRef]

30. Clinical Guidelines on the Identification, Evaluation, and Treatment of Overweight and Obesity in Adults-The Evidence Report. National Institutes of Health; National Heart, Lung, and Blood Institute: Bethesda, MD, USA, 1998; Volume 6 (Suppl. 2), pp. 51S-209S.

31. Population Structure and Ageing-Statistics Explained. Available online: https://ec.europa.eu/eurostat/ statistics-explained/index.php?title=Population_structure_and_ageing\#Median_age_is_highest_in_Italy (accessed on 25 April 2020).

32. Chung, M.L.; Lennie, T.A.; Mudd-Martin, G.; Moser, D.K. Adherence to the Low Sodium Diet in Patients with Heart Failure is Best When Family Members Also Follow the Diet: A Multicenter Observational Study. J. Cardiovasc Nurs. 2015, 30, 44-50. [CrossRef] 
33. Ayele, A.A.; Emiru, Y.K.; Tiruneh, S.A.; Ayele, B.A.; Gebremariam, A.D.; Tegegn, H.G. Level of adherence to dietary recommendations and barriers among type 2 diabetic patients: A cross-sectional study in an Ethiopian hospital. Clin. Diabetes Endocrinol. 2018, 4, 21. [CrossRef] [PubMed]

34. Mirahmadizadeh, A.; Khorshidsavar, H.; Seif, M.; Sharifi, M.H. Adherence to Medication, Diet and Physical Activity and the Associated Factors Amongst Patients with Type 2 Diabetes. Diabetes 2020, 11, 479-494. [CrossRef]

35. Karalis, D.G. A Review of Clinical Practice Guidelines for the Management of Hypertriglyceridemia: A Focus on High Dose Omega-3 Fatty Acids. Adv. Ther. 2017, 34, 300-323. [CrossRef] [PubMed]

36. Kolovou, G.D.; Anagnostopoulou, K.K.; Damaskos, D.S.; Bilianou, H.I.; Mihas, C.; Milionis, H.J.; Kostakou, P.M.; Cokkinos, D.V. Gender differences in the lipid profile of dyslipidemic subjects. Eur. J. Intern. Med. 2009, 20, 145-151. [CrossRef] [PubMed]

37. da Silva, P.M.; Aguiar, C.; Morais, J. DISGEN-LIPID study Investigators Suboptimal lipid levels in clinical practice among Portuguese adults with dyslipidemia under lipid-lowering therapy: Data from the DISGEN-LIPID study. Rev. Port. Cardiol. 2019, 38, 559-569. [CrossRef]

38. Tan, N.C.; Koh, E.Y.L.; Goh, C.C.; Goh, P.S.C.; Koh, K.H. A cross-sectional study of gender differences in lifestyle behavior and usage of medications among community-dwelling Asians towards achieving their LDL-Cholesterol treatment goals. Proc. Singap. Healthc. 2017, 26, 158-165. [CrossRef]

39. Wang, H.; Peng, D.-Q. New insights into the mechanism of low high-density lipoprotein cholesterol in obesity. Lipids Health Dis 2011, 10, 176. [CrossRef]

40. Wakabayashi, I.; Daimon, T. Associations between hypo-HDL cholesterolemia and cardiometabolic risk factors in middle-aged men and women: Independence of habitual alcohol drinking, smoking and regular exercise. Obes Res. Clin. Pr. 2017, 11, 324-334. [CrossRef]

41. Merianos, A.L.; Jandarov, R.A.; Khoury, J.C.; Mahabee-Gittens, E.M. Tobacco Smoke Exposure Association with Lipid Profiles and Adiposity Among U.S. Adolescents. J. Adolesc Health 2018, 62, 463-470. [CrossRef]

42. Becker, D.J.; Gordon, R.Y.; Halbert, S.C.; French, B.; Morris, P.B.; Rader, D.J. Red yeast rice for dyslipidemia in statin-intolerant patients: A randomized trial. Ann. Intern. Med. 2009, 150, 830-839. [CrossRef]

43. Bogsrud, M.P.; Ose, L.; Langslet, G.; Ottestad, I.; Strøm, E.C.; Hagve, T.-A.; Retterstøl, K. HypoCol (red yeast rice) lowers plasma cholesterol—A randomized placebo controlled study. Scand. Cardiovasc. J. 2010, 44, 197-200. [CrossRef]

44. Ogier, N.; Amiot, M.-J.; Georgé, S.; Maillot, M.; Mallmann, C.; Maraninchi, M.; Morange, S.; Lescuyer, J.-F.; Peltier, S.L.; Cardinault, N. LDL-cholesterol-lowering effect of a dietary supplement with plant extracts in subjects with moderate hypercholesterolemia. Eur. J. Nutr. 2013, 52, 547-557. [CrossRef]

45. Barrat, E.; Zaïr, Y.; Ogier, N.; Housez, B.; Vergara, C.; Maudet, C.; Lescuyer, J.-F.; Bard, J.-M.; Carpentier, Y.A.; Cazaubiel, M.; et al. A combined natural supplement lowers LDL cholesterol in subjects with moderate untreated hypercholesterolemia: A randomized placebo-controlled trial. Int J. Food Sci. Nutr. 2013, 64, 882-889. [CrossRef]

46. Barrat, E.; Zaïr, Y.; Sirvent, P.; Chauveau, P.; Maudet, C.; Housez, B.; Derbord, E.; Lescuyer, J.-F.; Bard, J.-M.; Cazaubiel, M.; et al. Effect on LDL-cholesterol of a large dose of a dietary supplement with plant extracts in subjects with untreated moderate hypercholesterolaemia: A randomised, double-blind, placebo-controlled study. Eur. J. Nutr. 2013, 52, 1843-1852. [CrossRef] [PubMed]

47. Higashikawa, F.; Noda, M.; Awaya, T.; Ushijima, M.; Sugiyama, M. Reduction of serum lipids by the intake of the extract of garlic fermented with Monascus pilosus: A randomized, double-blind, placebo-controlled clinical trial. Clin. Nutr. 2012, 31, 261-266. [CrossRef] [PubMed]

48. Barrios, V.; Escobar, C.; Cicero, A.F.G.; Burke, D.; Fasching, P.; Banach, M.; Bruckert, E. A nutraceutical approach (Armolipid Plus) to reduce total and LDL cholesterol in individuals with mild to moderate dyslipidemia: Review of the clinical evidence. Atheroscler. Suppl. 2017, 24, 1-15. [CrossRef] [PubMed]

49. Formisano, E.; Pasta, A.; Cremonini, A.L.; Favari, E.; Ronca, A.; Carbone, F.; Semino, T.; Di Pierro, F.; Sukkar, S.G.; Pisciotta, L. Efficacy of Nutraceutical Combination of Monacolin K, Berberine, and Silymarin on Lipid Profile and PCSK9 Plasma Level in a Cohort of Hypercholesterolemic Patients. J. Med. Food 2019. [CrossRef] [PubMed]

50. Adorni, M.P.; Ferri, N.; Marchianò, S.; Trimarco, V.; Rozza, F.; Izzo, R.; Bernini, F.; Zimetti, F. Effect of a novel nutraceutical combination on serum lipoprotein functional profile and circulating PCSK9. Clin. Risk Manag. 2017, 13, 1555-1562. [CrossRef] [PubMed] 
51. Eslick, G.D.; Howe, P.R.C.; Smith, C.; Priest, R.; Bensoussan, A. Benefits of fish oil supplementation in hyperlipidemia: A systematic review and meta-analysis. Int. J. Cardiol. 2009, 136, 4-16. [CrossRef] [PubMed]

52. Abramson, J. Statins in Persons at Low Risk of Cardiovascular Disease. Am. Fam Physician 2017, 96.

53. Cholesterol Treatment Trialists' (CTT) Collaborators; Mihaylova, B.; Emberson, J.; Blackwell, L.; Keech, A.; Simes, J.; Barnes, E.H.; Voysey, M.; Gray, A.; Collins, R. The effects of lowering LDL cholesterol with statin therapy in people at low risk of vascular disease: Meta-analysis of individual data from 27 randomised trials. Lancet 2012, 380, 581-590. [CrossRef]

54. Ridker, P.M.; Pradhan, A.; MacFadyen, J.G.; Libby, P.; Glynn, R.J. Cardiovascular benefits and diabetes risks of statin therapy in primary prevention: An analysis from the JUPITER trial. Lancet 2012, 380, 565-571. [CrossRef]

55. Ramkumar, S.; Raghunath, A.; Raghunath, S. Statin Therapy: Review of Safety and Potential Side Effects. Acta Cardiol. Sin. 2016, 32, 631-639. [CrossRef]

56. Pedan, A.; Varasteh, L.; Schneeweiss, S. Analysis of factors associated with statin adherence in a hierarchical model considering physician, pharmacy, patient, and prescription characteristics. J. Manag. Care Pharm. 2007, 13, 487-496. [CrossRef]

(C) 2020 by the authors. Licensee MDPI, Basel, Switzerland. This article is an open access article distributed under the terms and conditions of the Creative Commons Attribution (CC BY) license (http://creativecommons.org/licenses/by/4.0/). 